\begin{tabular}{|r|l|}
\hline \multicolumn{2}{|c|}{ Statistica Sinica Preprint No: SS-2021-0354 } \\
\hline Title & $\begin{array}{l}\text { Semiparametric Causal Mediation Analysis with } \\
\text { Unmeasured Mediator-Outcome Confounding }\end{array}$ \\
\hline Manuscript ID & SS-2021-0354 \\
\hline URL & http://www.stat.sinica.edu.tw/statistica/ \\
\hline DOI & $10.5705 /$ ss.202021.0354 \\
\hline Complete List of Authors & $\begin{array}{l}\text { BaoLuo Sun and } \\
\text { Ting Ye }\end{array}$ \\
\hline Corresponding Author & BaoLuo Sun \\
\hline E-mail & stasb@nus.edu.sg \\
\hline Notice: Accepted version subject to English editing.
\end{tabular}


Statistica Sinica

\title{
Semiparametric Causal Mediation Analysis with Unmeasured Mediator-Outcome Confounding
}

\author{
BaoLuo Sun* and and Ting $\mathrm{Ye}^{\dagger}$ \\ * Department of Statistics and Data Science, \\ National University of Singapore \\ ${ }^{\dagger}$ Department of Biostatistics, \\ University of Washington
}

Abstract:

Although the exposure can be randomly assigned in studies of mediation effects, any form of direct intervention on the mediator is often infeasible. As a result, unmeasured mediator-outcome confounding can seldom be ruled out. We propose semiparametric identification of natural direct and indirect effects in the presence of unmeasured mediator-outcome confounding by leveraging heteroskedasticity restrictions on the observed data law. For inference, we develop semiparametric estimators that remain consistent under partial misspecifications of the observed data model. We illustrate the proposed estimators through both simulations and an application to evaluate the effect of self-efficacy on fatigue among health care workers during the COVID-19 outbreak.

Key words and phrases: Causal Inference, multiple robustness, natural direct 
effect; natural indirect effect; unmeasured confounding.

\section{Introduction}

It is often of interest in the health and social sciences to investigate not only the total effect of a point exposure $A$ on an outcome $Y$, but also the direct and indirect effects operating through a given post-exposure mediating variable $M$. Since the seminal work of Baron and Kenny (1986) in the context of linear structural equation models, the notions of natural direct effect (NDE) and natural indirect effect (NIE) have been formalized in the context of a binary exposure under the potential outcomes framework Robins and Greenland, 1992; Pearl, 2001). The NDE and NIE are particularly useful for understanding the causal mediation mechanism as the sum of these two effects is the average treatment effect of $A$ on $Y$. Under the sequential ignorability assumption of no unmeasured confounding for the $A-M, A-Y$ and $M-Y$ relationships (See Section 2 for a more formal treatment), the NDE and NIE may be nonparametrically identified from the observed data distribution based on the so-called mediation formula (Pearl, 2001; VanderWeele and Vansteelandt, 2009; Imai et al., 2010b; Tchetgen Tchetgen and Shpitser, 2014; VanderWeele, 2015). Sequential ignorability is often stated as a conditional version within strata of a set of measured baseline covariates $X$ not affected by the exposure, in the hope that no residual 
unmeasured confounding remains within strata of the measured covariates.

A fully parametric approach to evaluating the mediation formula typically entails specifying models for both $E(Y \mid M, A, X)$ and $E(M \mid A, X)$ under appropriate link functions (VanderWeele and Vansteelandt, 2009, VanderWeele, 2015), which may be sensitive to model misspecifications. On the other hand, nonparametric inference yields multiply robust estimators which remain consistent and asymptotically normal (CAN) within various strict subsets of the observed data model, which includes the conditional densities $f(M \mid A, X)$ or $f(A \mid X)$ (Tchetgen Tchetgen and Shpitser, 2014). As a compromise between the fully parametric and nonparametric approaches, consider the following semiparametric partially linear outcome and mediator models indexed by $\theta=\left(\theta_{1}, \theta_{2}, \theta_{3}\right)^{\mathrm{T}} \in \mathbb{R}^{3}$,

$$
\begin{gathered}
E\left(Y \mid M, A, X ; \theta_{1}, \theta_{2}, g\right)=\theta_{1} M+\theta_{2} A+g(X) \\
E\left(M \mid A, X ; \theta_{3}, h\right)=\theta_{3} A+h(X)
\end{gathered}
$$

where the confounding effects of the measured covariates on the outcome and mediator are respectively encoded by the functions $g(\cdot)$ and $h(\cdot)$, which remain unspecified. If $X$ is sufficiently rich so that sequential ignorability holds, then $\theta_{2}$ and $\theta_{1} \theta_{3}$ respectively capture the NDE and NIE per unit change in the exposure (Hines et al., 2021). Let $\pi(X) \equiv E(A \mid X)$ denote the treatment propensity score and $O=(Y, A, M, X)$ the observed data. 
For estimation of $\theta$, Hines et al. (2021) considered the $3 \times 1$ vector estimating function $\varphi(O ; \theta, \pi, g, h)$ with components

$$
\begin{aligned}
& \varphi_{1}(O ; \theta, \pi, g)=\{A-\pi(X)\}\left\{Y-\theta_{1} M-\theta_{2} A-g(X)\right\} \\
& \varphi_{2}(O ; \theta, h, g)=\left\{M-\theta_{3} A-h(X)\right\}\left\{Y-\theta_{1} M-\theta_{2} A-g(X)\right\} \\
& \varphi_{3}(O ; \theta, \pi, h)=\{A-\pi(X)\}\left\{M-\theta_{3} A-h(X)\right\} .
\end{aligned}
$$

An augmented G-estimator (Robins, 1994) of $\theta$ may be constructed as the solution to the empirical moment condition

$$
n^{-1} \sum_{i=1}^{n} \varphi\left(O_{i} ; \theta, \hat{\pi}, \hat{g}, \hat{h}\right)=0
$$

where $(\hat{\pi}, \hat{g}, \hat{h})$ is a first-stage estimator of the nuisance parameters under user-specified parametric models. Provided any pair of nuisance parameters in $\{\pi(x), g(x), h(x)\}$ is correctly modeled, Hines et al. (2021) showed that the resulting augmented G-estimator is CAN for the true value of $\theta$ defined under partially linear model (1.1).

\subsection{Motivation and related work}

Although in principle one can rule out unmeasured confounding of the $A-M$ and $A-Y$ relationships by design when the exposure assignment is randomized (possibly within strata of a known set of measured baseline covariates), it is often infeasible to directly manipulate the mediator. As a result, numerous researchers have developed sensitivity analysis (Imai et al., 
2010a; VanderWeele, 2010; Tchetgen Tchetgen and Shpitser, 2012; Ding and

Vanderweele, 2016) and partial identification approaches (Sjölander, 2009,

Robins and Richardson, 2010a) to assess the impact of departures from the no unmeasured $M-Y$ confounding assumption. Identification of causal mediation mechanisms under unmeasured $M-Y$ confounding can sometimes be achieved through the principal stratification approach Gallop et al. 2009, Mattei and Mealli, 2011) or by leveraging ancillary variables that satisfy certain exclusion restrictions (Imai et al., 2013; Burgess et al., 2015; Frölich and Huber, 2017). Another major strand of work in the health sciences uses baseline covariates interacted with random exposure assignment as instrumental variables for the effect of the mediator on the outcome (Ten Have et al., 2007; Dunn and Bentall, 2007; Albert, 2008; Small, 2012; Zheng and Zhou, 2015); see also the commentary by Ogburn (2012).

Recently, there has been a growing interest in econometrics and health sciences to use higher-order moment restrictions as a source of identification in linear structural models without exclusion restrictions (Rigobon, 2003, Klein and Vella, 2010; Lewbel, 2012; Tchetgen Tchetgen et al., 2020). To the best of our knowledge, the work of Fulcher et al. (2019) is the first to extend this identification framework to causal mediation analysis with unmeasured $M-Y$ confounding. They considered identification and estimation 
of the NIE under structural assumptions which imply the semiparametric partially linear model

$$
\begin{gathered}
E\left(Y \mid M, A, X, U ; \theta_{1}, \theta_{2}, g\right)=\theta_{1} M+\theta_{2} A+g(X, U) \\
E\left(M \mid A, X, U ; \theta_{3}, h\right)=\theta_{3} A+h(X, U)
\end{gathered}
$$

where $U$ is a set of unmeasured baseline covariates, not affected by the exposure, that confounds the $M-Y$ relationship. Under further assumptions to be formalized in Section 2, the parameters $\theta_{1} \theta_{3}$ and $\theta_{2}$ encode the NIE and NDE per unit increase in the exposure respectively, which provide useful summary of the mediation effects. The unspecified functions $g(\cdot)$ and $h(\cdot)$ now encode the confounding effects of both measured and unmeasured covariates on the outcome and mediator, respectively. In this paper, we build upon the results of Fulcher et al. (2019) and establish identification of $\theta=\left(\theta_{1}, \theta_{2}, \theta_{3}\right)^{\mathrm{T}}$ (and hence both the NDE and NIE) under the partially linear model (1.3). Furthermore, similar to Hines et al. (2021), we propose augmented G-estimators that remain CAN for the true value of $\theta$ defined by 1.3 if any one of three strict subsets of the nuisance parameters lie in user-specified parametric models, including one in which the parametric models for the nuisance parameters considered by Fulcher et al. (2019) are correctly specified. This marks a significant improvement in robustness to model misspecification, which is especially useful in observational studies 
when $X$ contains numerous continuous components.

The rest of the paper is organized as follows. In section 2, we introduce the formal identification conditions for $\theta$ under partially linear model (1.3), and present multiply robust augmented G-estimation methods in section 3. We evaluate the finite-sample performance of these proposed methods through simulation studies in section 4 and illustrate the approach with a real data example in section 5. We explore several possible extensions including allowing for $A-M$ interactions in the outcome model in section 6 before ending with a brief discussion in section 7 .

\section{Notation and assumptions}

We use the potential outcomes framework (Neyman, 1923; Rubin, 1974) to define the mediation effects of interest. Let $M_{a}$ denote the mediator value that would be observed had the exposure $A$ been set, possibly contrary to fact, to level $a$. Similarly, let $Y_{a, m}$ denote the potential outcome that would be observed had $A$ been set to level $a$, and $M$ to $m$. The population NDE and NIE of $A$ on $Y$ comparing two exposure levels $a, a^{\prime}$ are given by $\operatorname{NDE}\left(a, a^{\prime}\right) \equiv E\left(Y_{a, M_{a^{\prime}}}-Y_{a^{\prime}, M_{a^{\prime}}}\right)$ and $\operatorname{NIE}\left(a, a^{\prime}\right) \equiv E\left(Y_{a, M_{a}}-Y_{a, M_{a^{\prime}}}\right)$ respectively (VanderWeele, 2015). The NDE and NIE are particularly relevant for describing the underlying mechanism by which the exposure operates, as their sum equals the population total effect given by $E\left(Y_{a, M_{a}}-Y_{a^{\prime}, M_{a^{\prime}}}\right)$. 
Under the sequential ignorability assumption that for any $\left(a, a^{\prime}, m\right)$,

$$
Y_{a, m} \perp A\left|X, \quad M_{a} \perp A\right| X, \quad Y_{a, m} \perp M\left|(A, X), \quad Y_{a, m} \perp M_{a^{\prime}}\right| X,
$$

where $B \perp C \mid D$ indicates conditional independence of $B$ and $C$ given $D$ (Dawid, 1979), the mediation effects are nonparametrically identified from the observed data distribution as the functionals

$$
\begin{aligned}
\operatorname{NDE}\left(a, a^{\prime}\right) & =\iint\left\{E(Y \mid a, m, x)-E\left(Y \mid a^{\prime}, m, x\right)\right\} f\left(m \mid a^{\prime}, x\right) f(x) d m d x \\
\operatorname{NIE}\left(a, a^{\prime}\right) & =\iint E(Y \mid a, m, x)\left\{f(m \mid a, x)-f\left(m \mid a^{\prime}, x\right)\right\} f(x) d m d x
\end{aligned}
$$

for all $a, a^{\prime}$ (Pearl, 2001; Imai et al., 2010b; VanderWeele, 2015). Evaluation of 2.2 in conjunction with the partially linear model (1.1) yields $\operatorname{NDE}\left(a, a^{\prime}\right)=\theta_{2}\left(a-a^{\prime}\right)$ and $\operatorname{NIE}\left(a, a^{\prime}\right)=\theta_{1} \theta_{3}\left(a-a^{\prime}\right)$; hence the NDE and NIE are identified as long as $\theta$ is identified.

\subsection{Identification under unmeasured $M-Y$ confounding}

In practical settings it is often infeasible to randomize or intervene on the mediator. Because any unmeasured $M-Y$ confounding that can seldom be ruled out, we will assume that 2.1 only holds conditional on $(X, U)$, i.e. for any $\left(a, a^{\prime}, m\right)$,

$Y_{a, m} \perp A\left|(X, U), \quad M_{a} \perp A\right|(X, U), \quad Y_{a, m} \perp M\left|(A, X, U), \quad Y_{a, m} \perp M_{a^{\prime}}\right|(X, U)$. 
In addition, we will assume that the exposure is randomly assigned either by design or through some natural experiments, so that

$$
A \perp U \mid X
$$

Figure 1 depicts the causal diagram for such a scenario. Under the latent sequential ignorability assumption (2.3), it is straightward to verify that the mediation effects are now given by the functionals

$$
\begin{aligned}
\operatorname{NDE}\left(a, a^{\prime}\right) & =\iint\left\{E(Y \mid a, m, x, u)-E\left(Y \mid a^{\prime}, m, x, u\right)\right\} f\left(m \mid a^{\prime}, x, u\right) f(x, u) d m d x d u \\
\operatorname{NIE}\left(a, a^{\prime}\right) & =\iint E(Y \mid a, m, x, u)\left\{f(m \mid a, x, u)-f\left(m \mid a^{\prime}, x, u\right)\right\} f(x, u) d m d x d u .
\end{aligned}
$$

Evaluation of 2.5 in conjunction with the partially linear model (1.3) yields $\operatorname{NDE}\left(a, a^{\prime}\right)=\theta_{2}\left(a-a^{\prime}\right)$ and $\operatorname{NIE}\left(a, a^{\prime}\right)=\theta_{1} \theta_{3}\left(a-a^{\prime}\right)$, a result given in Fulcher et al. (2019). The partially linear model $(1.3)$ in conjunction with a randomized exposure that satisfies (2.4) yield the conditional mean independence restrictions

$$
\begin{gathered}
E\left\{Y-\theta_{1} M-\theta_{2} A \mid A, X\right\}=E\{g(X, U) \mid A, X\}=g^{*}(X) ; \\
E\left\{M-\theta_{3} A \mid A, X\right\}=E\{h(X, U) \mid A, X\}=h^{*}(X) .
\end{gathered}
$$

The main challenge with identification and estimation based on (2.6) is that there are two restrictions but three unknown parameters in $\theta$. If $U$ is null and thus $g(X, U)=g^{*}(X)$ almost surely, Hines et al. (2021) derived 
augmented G-estimators of $\theta$ based on $E\left\{Y-\theta_{1} M-\theta_{2} A \mid A, M, X\right\}=g^{*}(X)$

which is a stronger version of the first restriction in (2.6). However, this restriction fails to hold when $U$ is non-null, as $E\{g(X, U) \mid A, M, X\}$ will remain a function of $(A, M, X)$ due to collider bias at $M$ within strata of $X$ as shown in figure 1. In this paper we do not impose this restriction, and instead leverage the conditional covariance mean independence restriction

$$
\begin{aligned}
E\left[\left\{M-\theta_{3} A-h^{*}(X)\right\}\left\{Y-\theta_{1} M\right\} \mid A, X\right] & =E[\operatorname{cov}\{g(X, U), h(X, U)\} \mid A, X] \\
& =\rho(X)
\end{aligned}
$$

which holds under 1.3 and 2.4 . We summarize the observed data restrictions below.

Lemma 1. Under partially linear model (1.3) and a randomized exposure which satisfies (2.4), the conditional mean independence restriction

$$
E\left\{\psi\left(O ; \theta, h^{*}\right) \mid A, X\right\}=E\left\{\psi\left(O ; \theta, h^{*}\right) \mid X\right\}
$$

holds almost surely, where $\psi\left(O ; \theta, h^{*}\right)$ is a $3 \times 1$ vector function with components $\psi_{1}(O ; \theta)=\left\{Y-\theta_{1} M-\theta_{2} A\right\}, \psi_{2}\left(O ; \theta, h^{*}\right)=\left\{M-\theta_{3} A-h^{*}(X)\right\}\{Y-$ $\left.\theta_{1} M\right\}$ and $\psi_{3}(O ; \theta)=\left\{M-\theta_{3} A\right\}$.

For identification, we also require (2.7) to have a unique solution for $\theta$. This may be partly justified by the linearity of the first and third components of $\psi\left(O ; \theta, h^{*}\right)$. The second component is nonlinear in $\theta$ and therefore 
require higher moment restrictions for identification. Following Fulcher et al. (2019), we will assume that the observed data distribution satisfies the heteroscedasticity condition that for any pair of exposure values $\left(a, a^{\prime}\right)$,

$$
\operatorname{var}(M \mid A=a, X) \neq \operatorname{var}\left(M \mid A=a^{\prime}, X\right) \text { if } a \neq a^{\prime},
$$

almost surely. We recommend that the Breusch-Pagan test for heteroskedasticity (Breusch and Pagan, 1979) be performed prior to analysis using the proposed method. Condition 2.8 may be motivated from the linear structural equation (Pearl, 2000)

$$
M=\lambda(A, X, U, \epsilon)=\lambda_{0}(\epsilon) A+\lambda_{1}(X, U, \epsilon)
$$

where $\lambda_{0}(\cdot), \lambda_{1}(\cdot)$ are unspecified functions and $\epsilon$ is a latent error that satisfies $\epsilon \perp(A, X, U)$. We note that (2.9) implies the mediator partially linear model in 1.3. Let $\tilde{\lambda}_{0}(\epsilon) \equiv \lambda_{0}(\epsilon)-E\left\{\lambda_{0}(\epsilon)\right\}$ and $\tilde{\lambda}_{1}(X, U, \epsilon) \equiv \lambda_{1}(X, U, \epsilon)-$ $E\left\{\lambda_{1}(X, U, \epsilon) \mid X\right\}$. Then the conditional variance $\operatorname{var}(M \mid A, X)=E\left[\left\{\tilde{\lambda}_{0}(\epsilon) A+\right.\right.$ $\left.\left.\tilde{\lambda}_{1}(X, U, \epsilon)\right\}^{2} \mid A, X\right]$ depends on $A$ provided $\lambda_{0}(\epsilon)$ depends on the latent source of effect heterogeneity $\epsilon$. Therefore, condition (2.8) does not hold under (2.9) and when exposure has no effect on the mediator for any individual. It does hold when there is heterogeneous exposure effect on the mediator in the population, a scenario which is plausible under a variety 
of health and social sciences settings (Tchetgen Tchetgen et al., 2020) and which permits the average exposure effect on the mediator to be zero, i.e. $E\left\{\lambda_{0}(\epsilon)\right\}=0$.

Theorem 1. Under partially linear model (1.3) and assumptions (2.4) and (2.8), the parameter $\theta$ is identified as the unique solution to

$$
E\left\{\psi\left(O ; \theta, h^{*}\right) \mid A, X\right\}=E\left\{\psi\left(O ; \theta, h^{*}\right) \mid X\right\}
$$

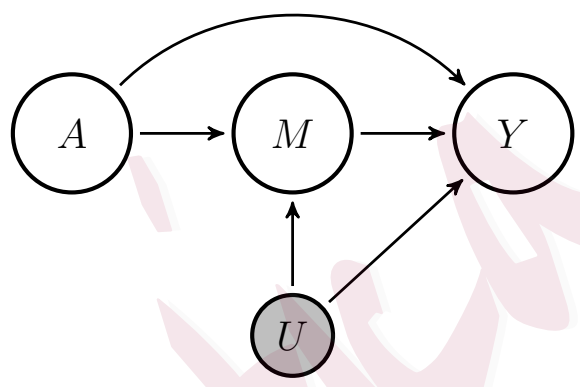

Figure 1: Causal diagram with unmeasured mediator-outcome confounding within strata of $X$.

\section{Semiparametric inference}

The conditional mean independence restriction (2.7) implies the following unconditional moment condition for $\theta$,

$$
0=E\left[\{A-\pi(X)\} \psi\left(O ; \theta, h^{*}\right)\right]
$$


which depends on the unknown nuisance parameters $\pi(x)$ and $h^{*}(x)$. In principle it is possible to estimate $\pi(x)$ and $h^{*}(x)$ nonparametrically under sufficient smoothness conditions (Ai and Chen, 2003; Newey and Powell, 2003). However, if $X$ contains numerous continuous components, the resulting estimators of $\theta$ typically exhibit poor finite sample behavior in moderately sized samples as the data are too sparse to conduct stratified estimation (Robins and Ritov, 1997). This setting is of particular relevance when the analyst considers a broad collection of covariates and their functional forms to render condition (2.4) plausible in observational studies.

As a remedy, following the augmented G-estimation approach (Robins, 1994), we propose estimators of $\theta$ which remain CAN if various strict subsets of the nuisance parameters $\eta=\left\{\pi(x), g^{*}(x), \rho(x), h^{*}(x)\right\}$ are correctly modeled. Towards this end, we derive the influence function of any regular and asymptotically linear estimator of $\theta$ based on 3.1 when $\left\{\pi(x), h^{*}(x)\right\}$ is estimated nonparametrically (Newey, 1994).

Theorem 2. The influence function of any regular and asymptotically linear estimator of $\theta$ when $\left\{\pi(x), h^{*}(x)\right\}$ is estimated nonparametrically is given by $-\Delta^{-1} \tilde{\varphi}(O ; \theta, \eta)$, where $\Delta \equiv E\left[\{A-\pi(X)\} \partial \psi\left(O ; \theta, h^{*}\right) / \partial \theta\right]$ and 
$\tilde{\varphi}(O ; \theta, \eta)$ is a $3 \times 1$ vector function with components

$$
\begin{aligned}
\tilde{\varphi}_{1}\left(O ; \theta, \pi, g^{*}\right)= & \{A-\pi(X)\}\left\{Y-\theta_{1} M-\theta_{2} A-g^{*}(X)\right\} \\
\tilde{\varphi}_{2}(O ; \theta, \eta)= & \{A-\pi(X)\}\left\{M-\theta_{3} A-h^{*}(X)\right\}\left\{Y-\theta_{1} M-\theta_{2} A-g^{*}(X)\right\} \\
& -\rho(X)
\end{aligned}
$$$$
\tilde{\varphi}_{3}\left(O ; \theta, \pi, h^{*}\right)=\{A-\pi(X)\}\left\{M-\theta_{3} A-h^{*}(X)\right\}
$$

The first and third components of $\tilde{\varphi}(O ; \theta, \eta)$ are the same as those in (1.2), although the second component differs due to its reliance on a different conditional mean independence restriction as discussed in Section 2.1. The proof of Theorem 2 in the Supplementary Material shows that $\tilde{\varphi}(O ; \theta, \eta)$ also equals to the original identifying moment condition (3.1) augmented with the nonparametric influence functions for estimation of $\left\{\pi(x), h^{*}(x)\right\}$. This yields the so-called orthogonal moment condition which is locally robust to the nuisance parameters on which it depends (Chernozhukov et al., 2020).

\subsection{Multiply robust estimation}

We propose semiparametric estimation of $\theta$ based on the estimating function $\tilde{\varphi}(O ; \theta, \eta)$ evaluated under the working parametric models

$$
\left\{\pi\left(x ; \eta_{1}\right), g^{*}\left(x ; \eta_{2}\right), \rho\left(x ; \eta_{3}\right), h^{*}\left(x ; \eta_{4}\right): \eta=\left(\eta_{1}^{\mathrm{T}}, \eta_{2}^{\mathrm{T}}, \eta_{3}^{\mathrm{T}}, \eta_{4}^{\mathrm{T}}\right)^{\mathrm{T}} \in \mathbb{R}^{\mathrm{q}}\right\}
$$

with $q<\infty$. Under regularity conditions the proposed semiparametric 
estimator of $\theta$ is shown to be CAN if one, but not necessarily more than one, of the following model assumptions hold:

$\mathcal{M}_{1}$ : The models for $\left\{\pi(x), g^{*}(x)\right\}$ are correct.

$\mathcal{M}_{2}$ : The models for $\left\{\pi(x), h^{*}(x)\right\}$ are correct.

$\mathcal{M}_{3}$ : The models for $\left\{g^{*}(x), \rho(x), h^{*}(x)\right\}$ are correct.

For estimation purposes, suppose that $\left(O_{1}, \ldots, O_{n}\right)$ are independent and identically distributed observations. Let $\hat{E}(\cdot)$ denote the empirical mean operator $\hat{E}\{h(O)\}=n^{-1} \sum_{i=1}^{n} h\left(O_{i}\right)$. We propose joint estimation of the parameters $(\theta, \eta)$, whereby the estimator $\hat{\theta}=\left(\hat{\theta}_{1}, \hat{\theta}_{2}, \hat{\theta}_{3}\right)^{\mathrm{T}}$ solves

$$
0=\hat{E}\{\tilde{\varphi}(O ; \theta, \hat{\eta}(\theta))\}
$$

with $\hat{\eta}(\theta)$ solving $0=\hat{E}\{\gamma(O ; \theta, \eta)\}$ for a fixed value of $\theta$, and $\gamma(O ; \theta, \eta)$ is a $q \times 1$ vector function with the components

$$
\begin{aligned}
\gamma_{1}(O ; \eta)= & \left\{\partial \pi\left(X ; \eta_{1}\right) / \partial \eta_{1}\right\}^{\mathrm{T}}\left\{A-\pi\left(X ; \eta_{1}\right)\right\} \\
\gamma_{2}(O ; \theta, \eta)= & \left\{\partial g^{*}\left(X ; \eta_{2}\right) / \partial \eta_{2}\right\}^{\mathrm{T}}\left\{Y-\theta_{1} M-\theta_{2} A-g^{*}\left(X ; \eta_{2}\right)\right\} \\
\gamma_{3}(O ; \theta, \eta)= & \left\{\partial \rho\left(X ; \eta_{3}\right) / \partial \eta_{3}\right\}^{\mathrm{T}}\left[\left\{M-\theta_{3} A-h^{*}\left(X ; \eta_{4}\right)\right\} \times\right. \\
& \left.\left\{Y-\theta_{1} M-\theta_{2} A-g^{*}\left(X ; \eta_{2}\right)\right\}-\rho\left(X ; \eta_{3}\right)\right] \\
\gamma_{4}(O ; \theta, \eta)= & \left\{\partial h^{*}\left(X ; \eta_{4}\right) / \partial \eta_{4}\right\}^{\mathrm{T}}\left\{M-\theta_{3} A-h^{*}\left(X ; \eta_{4}\right)\right\} .
\end{aligned}
$$


Lemma 2. Let $\theta^{\dagger}$ denote the unique solution to (3.1). Then under regularity conditions stated in the Appendix, $n^{1 / 2}\left(\hat{\theta}-\theta^{\dagger}\right) \stackrel{d}{\rightarrow} \mathcal{N}(0, \Sigma)$ as $n \rightarrow \infty$ in the union model $\left\{\cup_{j=1}^{3} \mathcal{M}_{j}\right\}$ (multiple robustness), where

$$
\Sigma=E\left(\left[E\left\{\left.\frac{\partial}{\partial \theta} \tilde{\Phi}\left(O ; \theta, \bar{\eta}\left(\theta^{\dagger}\right)\right)\right|_{\theta=\theta^{\dagger}}\right\}^{-1} \tilde{\Phi}\left(O ; \theta^{\dagger}, \bar{\eta}\left(\theta^{\dagger}\right)\right)\right]^{\otimes 2}\right)
$$

$\bar{\eta}(\theta)$ denotes the probability limit of $\hat{\eta}(\theta)$ and

$\tilde{\Phi}(O ; \theta, \eta)=\tilde{\varphi}(O ; \theta, \eta)-E\left\{\frac{\partial}{\partial \eta} \tilde{\varphi}(O ; \theta, \eta)\right\} E\left\{\frac{\partial}{\partial \eta} \gamma(O ; \theta, \eta)\right\}^{-1} \gamma(O ; \theta, \eta)$

As pointed out by a reviewer, $E\left\{\partial \tilde{\varphi}\left(O ; \theta^{\dagger}, \eta\right) /\left.\partial \eta\right|_{\eta=\bar{\eta}\left(\theta^{\dagger}\right)}\right\}=0$ at the intersection submodel $\left\{\cap_{j=1}^{3} \mathcal{M}_{j}\right\}$ where all the working models for the nuisance parameters are correctly specified, so that estimation of $\eta$ has no first order impact on the asymptotic variance of $\hat{\theta}$. This simplification does not hold in general when one or more of the working models are misspecified (Vermeulen and Vansteelandt, 2015). For inference, a consistent estimator $\hat{\Sigma}$ of $\Sigma$ may be constructed by replacing all expected values with empirical means evaluated at $\hat{\theta}$ and $\hat{\eta}(\hat{\theta})$. Then a $95 \%$ Wald confidence interval for the NDE per unit change in the exposure is found by calculating $\hat{\theta}_{2} \pm 1.96 \hat{\sigma}_{2}$, where $\hat{\sigma}_{2}$ is the square root of the $2^{\text {nd }}$ component of the diagonal of $n^{-1} \hat{\Sigma}$. Similar inference for the NIE per unit change in the exposure may be carried out using the multivariate delta method. Alternatively, nonparametric bootstrap may also be used to obtain estimates of $\Sigma$. 
Remark 1. As the nuisance parameters in $\mathcal{M}_{k}, k=1,2,3$ are variation independent, the proposed estimation framework provides the analyst with three genuine opportunities, instead of one, to obtain valid inferences about $\theta$ and functionals thereof, even under partial model misspecifications (Robins and Rotnitzky, 2001). Chernozhukov et al. (2018, 2020) established general regularity conditions for $n^{1 / 2}$-consistent estimation of finite dimensional parameters of interest based on orthogonal moment functions such as $\tilde{\varphi}(O ; \theta, \eta)$, even when the complexity of the nuisance parameter space for $\eta$ is no longer tractable by standard empirical process methods (e.g. Vapnik-Chervonenkis and Donsker classes). We plan to pursue this in future work.

Remark 2. The NIE estimator of $\theta_{1} \theta_{3}$ proposed by Fulcher et al. (2019) may be viewed as solving the empirical versions of only the second and third components in 3.1 , with both $\pi(x)$ and $h^{*}(x)$ estimated parametrically. It is clear that (3.1) does not have mean zero and therefore fails to identify $\left(\theta_{1}, \theta_{3}\right)$ when either or both parametric models for $\pi(x)$ and $h^{*}(x)$ are misspecified, i.e. the NIE estimator proposed by Fulcher et al. (2019) is CAN only in the semiparametric model $\mathcal{M}_{2}$. The proposed estimator $\hat{\theta}$ extends the estimation approach of Fulcher et al. (2019) in two main ways, by delivering $\sqrt{n}$-consistent inference about $\theta$ (and hence both the NDE 
and NIE) in the larger semiparametric union model $\left\{\cup_{j=1}^{3} \mathcal{M}_{j}\right\}$.

\section{Simulation study}

We perform simulations to study the pointwise properties of $\hat{\theta}$ and associated confidence intervals. We generate the baseline covariates $X_{1} \sim$ $\mathcal{N}(0,1)$ and $X_{2} \sim \mathcal{N}(0,1)$ independently, followed by

$$
\begin{gathered}
U \mid X \sim \mathcal{N}\left\{\mu=1+X_{1}-0.3 X_{2}, \sigma^{2}=\exp \left(-1.2+0.8 X_{1}-0.2 X_{2}\right)\right\} \\
A \mid X \sim \text { Bernoulli }\left[p=\left\{1+\exp \left(1-1.5 X_{1}+0.3 X_{2}\right)\right\}^{-1}\right] \\
M=1+(1.5+\epsilon) A+0.5 U, \quad Y=1+A+2 M+U
\end{gathered}
$$

where $U$ is an unmeasured factor that induce mediator-outcome dependence, and the latent effect heterogeneity generated independently as $\epsilon \sim$ $N(0,1)$ is introduced so that condition 2.8 holds. The true NDE and NIE for a unit change of exposure value are 1 and 3 respectively. Besides the proposed multiply robust estimator MR, we also implement the propensity score-based estimator PS of Fulcher et al. (2019) and the product of coefficients estimator BK of Baron and Kenny (1986) which does not account for unmeasured $M-Y$ confounding. We evaluate the estimators under the following four scenarios to investigate the impact of model misspecification for the nuisance parameters: (i) $\left\{\pi(x), g^{*}(x), \rho(x), h^{*}(x)\right\}$ are all correctly modeled; (ii) only $\left\{\pi(x), g^{*}(x)\right\}$ are correctly modeled; (iii) only $\left\{\pi(x), h^{*}(x)\right\}$ are correctly modeled, (iv) only $\left\{g^{*}(x), \rho(x), h^{*}(x)\right\}$ are correctly modeled 
and (v) none of the nuisance parameters are correctly modeled. Further details on model specification may be found in the Supplementary Material. A model is misspecified if the standardized versions of the transformed variables $\left[\exp \left(0.5 X_{1}\right), 10+X_{2} /\left\{1+\exp \left(X_{1}\right)\right\}\right]$ are used as regressors instead of $\left(X_{1}, X_{2}\right)$. Standard errors are obtained using the empirical sandwich estimator.

We simulate 1000 replicates with sample sizes $n=400,800$ for each scenario and summarize the results for estimation of NDE and NIE for a change of exposure value from 0 to 1 in Table 1. The estimators MR and PS perform similarly to each other in terms of absolute bias and coverage in scenarios (i) and (iii), but MR yields noticeably smaller absolute biases and better coverage than PS in scenarios (ii) and (iv) where either the model for $\pi(x)$ or $h^{*}(x)$ is misspecified. When none of the nuisance parameters are correctly modeled, all estimators show bias with coverage proportion below nominal value in the estimation of either NDE or NIE. In general PS is less efficient than MR, as the latter incorporates additional regression models that capture the associations between $(Y, M)$ and $(A, X)$. The estimator BK shows large bias and poor coverage across scenarios (i)-(v), in agreement with theory.

We also perform simulations with correctly specified models for the nui- 
sance parameters under two additional scenarios: (vi) weaker dependence of $\operatorname{var}(M \mid A, X)$ on $A$ as well as (vii) no unmeasured $M-Y$ confounding. For (vii) we compare the proposed approach with two competing methods under partially linear model (1.1), the product of coefficients estimator BK and the triply robust G-estimator TG of Hines et al. (2021). The simulation design and results for these two scenarios are included in the Supplementary Material. Compared to the results in scenario (i), the bias and variance of MR and PS increase in (vi), while their coverage remains close to nominal level. In scenario (vii), all estimators yield negligible bias and good coverage, but MR and PS have larger variance than BK and TG. 
Table 1: Summary of results for estimation of NDE and NIE for a change of exposure value from 0 to 1 . The

first and second rows' results for each estimator correspond to sample sizes $n=400,800$ respectively.

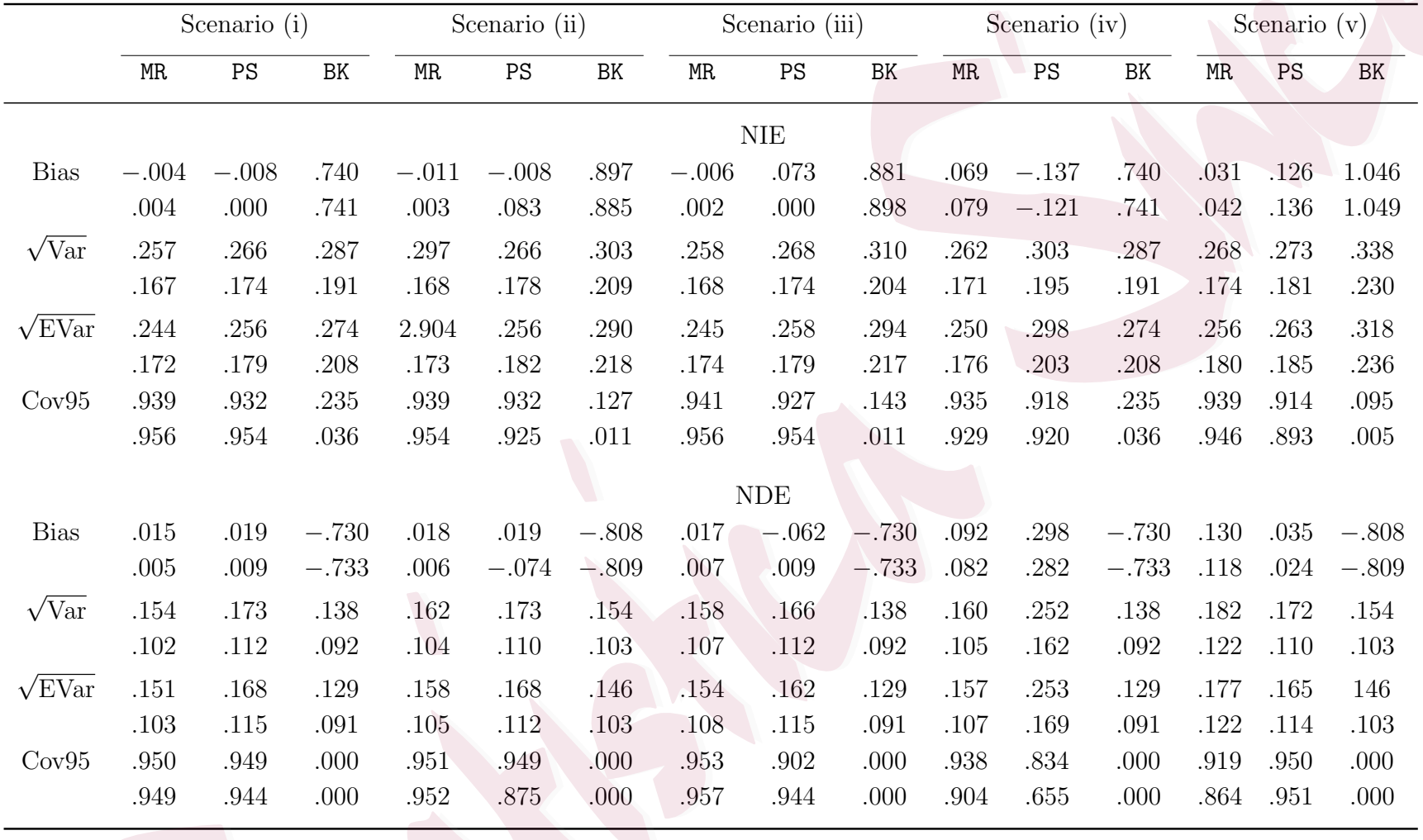

Note: Bias and $\sqrt{\operatorname{Var}}$ are the Monte Carlo bias and standard deviation of the point estimates, $\sqrt{\text { EVar }}$ is the square

root of the mean of the variance estimates and Cov95 is the coverage proportion of the $95 \%$ Wald confidence interval,

based on 1000 replicates. 


\section{Application}

We apply the proposed methods in reanalysing an observational study investigating the mediating effect of posttraumatic stress disorder (PTSD) symptoms in the association between self-efficacy and fatigue among health care workers during the COVID-19 outbreak (Hou et al., 2020). The crosssectional data was collected between March 13 and 20, 2020, from $n=$ 527 health care workers in Anqing City, Anhui Province, China, which borders Hubei province, the epicenter of the COVID-19 outbreak. We refer interested readers to Hou et al. (2020) for further details on the study design.

For this illustration, the continuous exposure $A$ is the standardized total score on the General Self-Efficacy Scale. We also consider the binary exposure $A$ which takes on value 1 if self-efficacy is above the sample median of total scores on the General Self-Efficacy Scale, and 0 otherwise. PTSD symptoms $(M)$ and fatigue $(Y)$ are standardized total scores on the PTSD Checklist-Civilian Version and 14-item Fatigue Scale respectively. The vector of observed baseline covariates $X$ consists of an intercept, age, gender, marital status, education level, work experience (in years) and seniority, as well as level of negative coping dichotomized at the sample median.

We specify the working models $\pi\left(x ; \eta_{1}\right)=\eta_{1}^{\mathrm{T}} x$ for continuous exposure 
or $\pi\left(x ; \eta_{1}\right)=\left\{1+\exp \left(-\eta_{1}^{\mathrm{T}} x\right)\right\}^{-1}$ for binary exposure, $g^{*}\left(x ; \eta_{2}\right)=\eta_{2}^{\mathrm{T}} x$, $\rho\left(x ; \eta_{3}\right)=\exp \left(\eta_{3}^{\mathrm{T}} x\right)$ and $h^{*}\left(x ; \eta_{4}\right)=\eta_{4}^{\mathrm{T}} x$. The main effects generalized linear models for the nuisance parameters are chosen due to their simplicity for illustration. In principle, the goodness-of-fit may be evaluated based on a generalized version of Akaike's information criterion Konishi and Kitagawa, 1996). Alternatively, one may also leverage the multiple robustness property in Lemma 2 for the purpose of model selection of nuisance parameters (Robins et al., 2020; Cui and Tchetgen Tchetgen, 2019; Sun et al. 2019). We acknowledge this limitation and defer model selection to future work. Due to the limited sample size and because negative coping $\left(X_{n c}\right)$ has been hypothesised as an important effect modifier of the exposure's effects on both the mediator and outcome (Hou et al., 2020), we further specify $\theta_{1}\left(x ; \beta_{1}\right)=\beta_{1}^{\mathrm{T}}\left(1, x_{n c}\right)^{\mathrm{T}}$ and $\theta_{3}\left(x ; \beta_{3}\right)=\beta_{3}^{\mathrm{T}}\left(1, x_{n c}\right)^{\mathrm{T}}$. The Breusch-Pagan test for heteroskedasticity (Breusch and Pagan, 1979) based on identical working models for the conditional mediator mean and variance yields p-values of $8.77 \times 10^{-7}$ and 0.04 for the continuous and binary exposure respectively, which indicates that the heteroscedasticity condition $(2.8)$ is plausible.

Table 2 shows various estimates of the NDE and NIE of self-efficacy on fatigue mediated though PTSD symptoms. With continuous exposure, the regression approach BK of Baron and Kenny (1986) yields a NIE estimate 
Table 2: Estimates $( \pm 1.96 \times$ standard error $)$ of NDE and NIE of self-efficacy on fatigue mediated though PTSD symptoms.

\begin{tabular}{|c|c|c|c|}
\hline & MR & PS & BK \\
\hline \multicolumn{4}{|c|}{ Continuous exposure } \\
\hline NDE & $-.352 \pm .119$ & $-.455 \pm .171$ & $-.234 \pm .083$ \\
\hline NIE & $-.042 \pm .084$ & $.062 \pm .130$ & $-.159 \pm .053$ \\
\hline \multicolumn{4}{|c|}{ Dichotomized exposure } \\
\hline NDE & $-.765 \pm .340$ & $-.748 \pm .332$ & $-.426 \pm .146$ \\
\hline NIE & $.066 \pm .308$ & $.049 \pm .297$ & $-.273 \pm .084$ \\
\hline
\end{tabular}

with $95 \%$ confidence interval $-.159 \pm .053$. This result suggests a significant mediating effect via PTSD symptoms in reducing fatigue, which is consistent with the original findings by Hou et al. (2020). The proposed approach MR yields a NIE estimate close to 0 , and the concomitant $95 \%$ confidence interval $-.042 \pm .084$ includes 0 . This suggests that we cannot rule out a null NIE after accounting for possible unmeasured common causes of PTSD and fatigue. Dichotomizing the exposure yields qualitatively similar results.

\section{Extensions}

\subsection{Exposure-mediator interaction}

In the presence of potential $A-M$ interaction in their effects on the 
outcome, we may consider the following partially linear models indexed by $\theta=\left(\theta_{1}, \theta_{2}, \zeta, \theta_{3}\right)^{\mathrm{T}} \in \mathbb{R}^{4}$

$$
\begin{gathered}
E\left(Y \mid M, A, X, U ; \theta_{1}, \theta_{2}, \zeta, g\right)=\theta_{1} M+\theta_{2} A+\zeta A M+g(X, U) \\
E\left(M \mid A, X, U ; \theta_{3}, h\right)=\theta_{3} A+h(X, U),
\end{gathered}
$$

where $\zeta$ is a scalar parameter encoding the interaction. If interaction is absent, so that $\zeta=0$, then (6.1) reduces to 1.3 . Evaluation of 2.2 in conjunction with 6.1 yields $\operatorname{NDE}\left(a, a^{\prime}\right)=\left(\theta_{2}+\zeta\left[\theta_{3} a^{\prime}+E\left\{h^{*}(X)\right\}\right]\right)\left(a-a^{\prime}\right)$ and $\operatorname{NIE}\left(a, a^{\prime}\right)=\theta_{3}\left(\theta_{1}+\zeta a\right)\left(a-a^{\prime}\right)$ (VanderWeele, 2015). Because the moment condition

$$
0=E\left[\omega(X)\{A-\pi(X)\} \psi\left(O ; \theta, h^{*}\right)\right]
$$

holds for an arbitrary $4 \times 1$ vector function $\omega(\cdot), \theta$ is identified via $(6.2)$ if

$$
E\left[\omega(X)\{A-\pi(X)\} \partial \psi\left(O ; \theta, h^{*}\right) / \partial \theta\right]
$$

is non-singular. A multiply robust estimator of $\theta$ may then be constructed based on the empirical version of the corresponding orthogonal moment condition. However, because of its dependence on $h^{*}(x)$, the proposed estimator of $\operatorname{NDE}\left(a, a^{\prime}\right)$ can only be doubly robust in the union model $\left\{\mathcal{M}_{2} \cup \mathcal{M}_{3}\right\}$

\subsection{Binary mediator}


The proposed semiparametric framework also extends to a binary mediator under the following log-linear model,

$$
\begin{aligned}
& E\left(Y \mid M, A, X, U ; \theta_{1}, \theta_{2}, g\right)=\theta_{1} M+\theta_{2} A+g(X, U) ; \\
& \log \left\{p\left(M=1 \mid A, X, U ; \theta_{3}, h\right)\right\}=\theta_{3} A+h(X, U) .
\end{aligned}
$$

Evaluation of (2.5) in conjunction with the log-linear model (6.3) yields $\operatorname{NDE}\left(a, a^{\prime}\right)=\theta_{2}\left(a-a^{\prime}\right)$ and $\operatorname{NIE}\left(a, a^{\prime}\right)=\theta_{1}\left\{\exp \left(\theta_{3} a\right)-\exp \left(\theta_{3} a^{\prime}\right)\right\} E\{\tilde{h}(X)\}$, where

$$
\tilde{h}(X)=E\left[M e^{-\theta_{3} A} \mid X\right]=E[\exp \{h(X, U)\} \mid X]
$$

Under the log-linear model $(6.3)$ and a randomized exposure which satisfies (2.4), the conditional mean independence restriction

$$
E\{\tilde{\psi}(O ; \theta, \tilde{h}) \mid A, X\}=E\{\tilde{\psi}(O ; \theta, \tilde{h}) \mid X\}
$$

holds almost surely, where $\tilde{\psi}(O ; \theta, \tilde{h})$ is a $3 \times 1$ vector function with components $\tilde{\psi}_{1}(O ; \theta)=\left\{Y-\theta_{1} M-\theta_{2} A\right\}, \tilde{\psi}_{2}(O ; \theta, \tilde{h})=\left\{Y-\theta_{1} M\right\}\left\{M \exp \left(-\theta_{3} A\right)-\right.$ $\tilde{h}(X)\}$ and $\tilde{\psi}_{3}(O ; \theta)=\left\{M \exp \left(-\theta_{3} A\right)\right\}$. Augmented G-estimation of $\theta$ may proceed based on identifying restriction (6.4), using methods analogous to those described in section 3. For binary outcome, a direct extension of existing methods to identify NDE and NIE on the risk ratio scale generally require the conditional density $f(M \mid A, X, U)$ to be normal with constant 
variance (VanderWeele and Vansteelandt, 2010; Valeri and VanderWeele, 2013: VanderWeele, 2015), in which case the heteroscedasticity condition (2.8) fails to hold. A possible direction is to identify and estimate NDE and NIE on the risk difference scale under the proposed framework.

\section{Discussions}

Unmeasured $M-Y$ confounding is particularly pernicious for credible causal mediation analysis in the health and social sciences, as the mediator can seldom be directly manipulated. The main contribution of this paper is a robust inference framework for the NDE and NIE under unmeasured $M-Y$ confounding in partially linear models by leveraging heteroscedasticity of $M$ with respect to $A$, a condition which is empirically testable. It should be pointed out here that the fourth condition $Y_{a, m} \perp M_{a^{*}} \mid(X, U)$ in (2.3) cannot be guaranteed through experimental interventions, even if we were able to randomize both the exposure and the mediator (Didelez et al. 2006; Imai et al., 2013; Robins and Richardson, 2010b). In the absence of exposure-induced confounding, dropping the fourth condition from (2.3) imbues the functionals in 2.5 with alternative causal interpretations as interventional analogues of the NDE and NIE (VanderWeele et al., 2014; VanderWeele, 2015). Because all our results in this paper concern identification and estimation of the functionals in 2.5 , they can be readily applied 
under this alternative interpretation. We conjecture that (2.7) represents all the observed data conditional mean restrictions under the partially linear structural model (1.3) and independence assumption 2.4. The number of observed data restrictions may potentially increase under stronger structural assumptions, e.g. by imposing restrictions on the structural distributions $f(Y \mid M, A, X, U)$ and $f(M \mid A, X, U)$. The proposed framework can also be extended in several other important directions, including mediation analysis with survival data and multiple mediators (Lin et al., 2017; Huang and Yang, 2017) under unmeasured mediator-outcome confounding, which we plan to pursue in future research.

\section{Supplementary Materials}

Supplementary material available includes the proof of Lemmas and Theorems and additional simulation results.

\section{Acknowledgments}

BaoLuo Sun is supported by the National University of Singapore StartUp Grant (R-155-000-203-133). We thank the Associate Editor and two reviewers for constructive comments leading to improvement of the article. We thank Eric Tchetgen Tchetgen and Xu Shi for helpful suggestions on a previous version of the article. 
REFERENCES

\section{References}

Ai, C. and Chen, X. (2003). Efficient estimation of models with conditional moment restrictions containing unknown functions. Econometrica, 71(6):1795-1843.

Albert, J. M. (2008). Mediation analysis via potential outcomes models. Statistics in medicine, 27(8):1282-1304.

Baron, R. M. and Kenny, D. A. (1986). The moderator-mediator variable distinction in social psychological research: Conceptual, strategic, and statistical considerations. Journal of personality and social psychology, 51(6):1173.

Breusch, T. S. and Pagan, A. R. (1979). A simple test for heteroscedasticity and random coefficient variation. Econometrica: Journal of the Econometric Society, pages 1287-1294.

Burgess, S., Daniel, R. M., Butterworth, A. S., Thompson, S. G., and Consortium, E.-I. (2015). Network mendelian randomization: using genetic variants as instrumental variables to investigate mediation in causal pathways. International journal of epidemiology, 44(2):484495.

Chernozhukov, V., Chetverikov, D., Demirer, M., Duflo, E., Hansen, C., Newey, W., and Robins, J. (2018). Double/debiased machine learning for treatment and structural parameters. The Econometrics Journal, 21(1):C1-C68.

Chernozhukov, V., Escanciano, J. C., Ichimura, H., Newey, W. K., and Robins, J. M. (2020). Locally robust semiparametric estimation. 
REFERENCES

Cui, Y. and Tchetgen Tchetgen, E. (2019). Selective machine learning of doubly robust functionals. arXiv preprint arXiv:1911.02029.

Dawid, A. P. (1979). Conditional independence in statistical theory. Journal of the Royal Statistical Society: Series B (Methodological), 41(1):1-15.

Didelez, V., Dawid, A. P., and Geneletti, S. (2006). Direct and indirect effects of sequential treatments. In Proceedings of the Twenty-Second Conference on Uncertainty in Artificial Intelligence, UAI'06, pages 138-146, Arlington, Virginia, USA. AUAI Press.

Ding, P. and Vanderweele, T. J. (2016). Sharp sensitivity bounds for mediation under unmeasured mediator-outcome confounding. Biometrika, 103(2):483-490.

Dunn, G. and Bentall, R. (2007). Modelling treatment-effect heterogeneity in randomized controlled trials of complex interventions (psychological treatments). Statistics in Medicine, 26(26):4719-4745.

Frölich, M. and Huber, M. (2017). Direct and indirect treatment effects-causal chains and mediation analysis with instrumental variables. Journal of the Royal Statistical Society: Series B (Statistical Methodology), 79(5):1645-1666.

Fulcher, I. R., Shi, X., and Tchetgen Tchetgen, E. J. (2019). Estimation of natural indirect effects robust to unmeasured confounding and mediator measurement error. Epidemiology, $30(6): 825-834$.

Gallop, R., Small, D. S., Lin, J. Y., Elliott, M. R., Joffe, M., and Ten Have, T. R. (2009). Mediation analysis with principal stratification. Statistics in medicine, 28(7):1108-1130. 
REFERENCES

Hines, O., Vansteelandt, S., and Diaz-Ordaz, K. (2021). Robust inference for mediated effects in partially linear models. Psychometrika, pages 1-24.

Hou, T., Dong, W., Zhang, R., Song, X., Zhang, F., Cai, W., Liu, Y., and Deng, G. (2020). Selfefficacy and fatigue among health care workers during covid-19 outbreak: A moderated mediation model of posttraumatic stress disorder symptoms and negative coping. Preprint.

Huang, Y.-T. and Yang, H.-I. (2017). Causal mediation analysis of survival outcome with multiple mediators. Epidemiology (Cambridge, Mass.), 28(3):370.

Imai, K., Keele, L., and Tingley, D. (2010a). A general approach to causal mediation analysis. Psychological methods, 15(4):309.

Imai, K., Keele, L., and Yamamoto, T. (2010b). Identification, inference and sensitivity analysis for causal mediation effects. Statistical science, pages 51-71.

Imai, K., Tingley, D., and Yamamoto, T. (2013). Experimental designs for identifying causal mechanisms. Journal of the Royal Statistical Society: Series A (Statistics in Society), $176(1): 5-51$

Klein, R. and Vella, F. (2010). Estimating a class of triangular simultaneous equations models without exclusion restrictions. Journal of Econometrics, 154(2):154-164.

Konishi, S. and Kitagawa, G. (1996). Generalised information criteria in model selection. Biometrika, 83(4):875-890.

Lewbel, A. (2012). Using heteroscedasticity to identify and estimate mismeasured and endoge- 
REFERENCES

nous regressor models. Journal of Business \& Economic Statistics, 30(1):67-80.

Lin, S.-H., Young, J. G., Logan, R., and VanderWeele, T. J. (2017). Mediation analysis for a survival outcome with time-varying exposures, mediators, and confounders. Statistics in medicine, 36(26):4153-4166.

Mattei, A. and Mealli, F. (2011). Augmented designs to assess principal strata direct effects. Journal of the Royal Statistical Society: Series B (Statistical Methodology), 73(5):729-752.

Newey, W. K. (1994). The asymptotic variance of semiparametric estimators. Econometrica: Journal of the Econometric Society, pages 1349-1382.

Newey, W. K. and Powell, J. L. (2003). Instrumental variable estimation of nonparametric models. Econometrica, 71(5):1565-1578.

Neyman, J. (1923). Sur les applications de la théorie des probabilités aux experiences agricoles: Essai des principes. Roczniki Nauk Rolniczych, 10:1-51.

Ogburn, E. L. (2012). Commentary of "mediation analysis without sequential ignorability: using baseline covariates interacted with random assignment as instrumental variables". Journal of Statistical Research, 46:105-111.

Pearl, J. (2000). Causality: Models, Reasoning and Inference. Cambridge, UK: Cambridge University Press.

Pearl, J. (2001). Direct and indirect effects. In Proceedings of the Seventeenth Conference on Uncertainty in Artificial Intelligence, UAI'01, pages 411-420, San Francisco, CA, USA. 
REFERENCES

Morgan Kaufmann Publishers Inc.

Rigobon, R. (2003). Identification through heteroskedasticity. Review of Economics and Statistics, 85(4):777-792.

Robins, J., Sued, M., Lei-Gomez, Q., and Rotnitzky, A. (2020). Double-robust and efficient methods for estimating the causal effects of a binary treatment. arXiv preprint arXiv:2008.0050\%.

Robins, J. M. (1994). Correcting for non-compliance in randomized trials using structural nested mean models. Communications in Statistics-Theory and methods, 23(8):2379-2412.

Robins, J. M. and Greenland, S. (1992). Identifiability and exchangeability for direct and indirect effects. Epidemiology, pages 143-155.

Robins, J. M. and Richardson, T. (2010a). Alternative graphical causal models and the identification of direct effects. In Shrout, P., Keyes, K., and Ornstein, K., editors, Causality and Psychopathology: Finding the Determinants of Disorders and Their Cures, pages 103-58. Oxford University Press, Oxford, UK.

Robins, J. M. and Richardson, T. S. (2010b). Alternative graphical causal models and the identification of direct effects. Causality and psychopathology: Finding the determinants of disorders and their cures, pages 103-158.

Robins, J. M. and Ritov, Y. (1997). Toward a curse of dimensionality appropriate (coda) asymptotic theory for semi-parametric models. Statistics in medicine, 16(3):285-319. 
REFERENCES

Robins, J. M. and Rotnitzky, A. (2001). Comment on the bickel and kwon article, "inference for semiparametric models: Some questions and an answer". Statistica Sinica, 11(4):920-936.

Rubin, D. B. (1974). Estimating causal effects of treatments in randomized and nonrandomized studies. Journal of educational Psychology, 66(5):688.

Sjölander, A. (2009). Bounds on natural direct effects in the presence of confounded intermediate variables. Statistics in Medicine, 28(4):558-571.

Small, D. S. (2012). Mediation analysis without sequential ignorability: using baseline covariates interacted with random assignment as instrumental variables. Journal of Statistical Research, 46:91-103.

Sun, B., Cui, Y., and Tchetgen Tchetgen, E. (2019). Selective machine learning of the average treatment effect with an invalid instrumental variable. arXiv preprint arXiv:190\%.11882.

Tchetgen Tchetgen, E. J. and Shpitser, I. (2012). Semiparametric theory for causal mediation analysis: efficiency bounds, multiple robustness, and sensitivity analysis. Annals of statistics, 40(3):1816.

Tchetgen Tchetgen, E. J. and Shpitser, I. (2014). Estimation of a semiparametric natural direct effect model incorporating baseline covariates. Biometrika, 101(4):849-864.

Tchetgen Tchetgen, E. J., Sun, B., and Walter, S. (2020). The genius approach to robust mendelian randomization inference. Statistical Science, in press.

Ten Have, T. R., Joffe, M. M., Lynch, K. G., Brown, G. K., Maisto, S. A., and Beck, A. T. 
REFERENCES

(2007). Causal mediation analyses with rank preserving models. Biometrics, 63(3):926-934.

Valeri, L. and VanderWeele, T. J. (2013). Mediation analysis allowing for exposure-mediator interactions and causal interpretation: theoretical assumptions and implementation with sas and spss macros. Psychological methods, 18(2):137.

VanderWeele, T. J. (2010). Bias formulas for sensitivity analysis for direct and indirect effects. Epidemiology (Cambridge, Mass.), 21(4):540.

VanderWeele, T. J. (2015). Explanation in causal inference: methods for mediation and interaction. Oxford University Press.

VanderWeele, T. J. and Vansteelandt, S. (2009). Conceptual issues concerning mediation, interventions and composition. Statistics and its Interface, 2(4):457-468.

VanderWeele, T. J. and Vansteelandt, S. (2010). Odds ratios for mediation analysis for a dichotomous outcome. American journal of epidemiology, 172(12):1339-1348.

VanderWeele, T. J., Vansteelandt, S., and Robins, J. M. (2014). Effect decomposition in the presence of an exposure-induced mediator-outcome confounder. Epidemiology (Cambridge, Mass.), 25(2):300.

Vermeulen, K. and Vansteelandt, S. (2015). Bias-reduced doubly robust estimation. Journal of the American Statistical Association, 110(511):1024-1036.

Zheng, C. and Zhou, X.-H. (2015). Causal mediation analysis in the multilevel intervention and multicomponent mediator case. Journal of the Royal Statistical Society: Series B: 
REFERENCES

Statistical Methodology, pages 581-615.

Department of Statistics and Data Science, National University of Singapore

E-mail: stasb@nus.edu.sg

Department of Biostatistics, University of Washington

E-mail: tingye1@uw.edu 